University of Wollongong

Research Online

Faculty of Social Sciences - Papers (Archive) Faculty of Arts, Social Sciences \& Humanities

2018

Pilot of an acceptance and commitment therapy and schema group intervention for mental health carer's interpersonal problems

Elly L. Quinlan

University of Wollongong, elly@uow.edu.au

Frank P. Deane

University of Wollongong, fdeane@uow.edu.au

Trevor P. Crowe

University of Wollongong, tcrowe@uow.edu.au

Follow this and additional works at: https://ro.uow.edu.au/sspapers

Part of the Education Commons, and the Social and Behavioral Sciences Commons

Research Online is the open access institutional repository for the University of Wollongong. For further information contact the UOW Library: research-pubs@uow.edu.au 


\title{
Pilot of an acceptance and commitment therapy and schema group intervention for mental health carer's interpersonal problems
}

\author{
Abstract \\ The term 'mental health carer' refers to any person who voluntarily provides ongoing care and assistance \\ to another person because of mental health issues (Carers Recognition Act, 2010). \\ Disciplines \\ Education | Social and Behavioral Sciences \\ Publication Details \\ Quinlan, E., Deane, F. \& Crowe, T. (2018). Pilot of an acceptance and commitment therapy and schema \\ group intervention for mental health carer's interpersonal problems. Journal of Contextual Behavioral \\ Science, 9 53-62.
}




\begin{abstract}
Mental health carers often have relationship difficulties with the person for whom they are caring and experience high rates of interpersonal problems compared to the general population. This study piloted a manualised 12-week group ACT and Schema intervention for mental health carers’ interpersonal problems, examining acceptability and preliminary effectiveness. This study had a mixed-methods design, with assessment booklets administer red at weeks 1, 6 and 12 of the program and focus groups conducted three months post. Participants comprised 24 mental health carers across five groups. Results indicated high attendance rates and positive perceptions of the intervention. Repeated measures ANOVAs revealed significant improvements in interpersonal problems, experiential avoidance, caregiving avoidance, mindfulness and wellbeing over time. Qualitative results highlighted themes of group process, reactivity, changes in emotion, acceptance of caregiving, communication, agency and connection. In conclusion, ACT and Schema group interventions show promise for mental health carers' interpersonal problems, however, larger controlled trials are required.
\end{abstract}




\section{Introduction}

The term 'mental health carer' refers to any person who voluntarily provides ongoing care and assistance to another person because of mental health issues (Carers Recognition Act, 2010). The last decade has seen increased focus on the complex interpersonal patterns that exist between carers and care receivers. This has been in response to advocacy by carers for recognition of the interpersonal aspects of their role (Wilkinson \& McAndrew, 2008; Henderson, 2001; Sadler \& McKevitt, 2013) and criticisms of the intrapersonal focus embedded in carer research, policy and service delivery (Chattoo \& Ahmad, 2008). Mental health carers often place great value on sustaining a comfortable and loving relationship with the person for whom they provide care (Gray, Seddon, Robinson, \& Roberts, 2009; Lawn \& McMahon, 2014; Spector, Charlesworth, Orrell, \& Marston, 2016), and such relationships are associated with increased carer coping and resiliency (Wadham, Simpson, Rust, \& Murray, 2016), increased wellbeing (Braithwaite, 2000), and decreased stress and depression (Oyebode, 2003). However, a recent study of 147 mental health carers indicated nearly one in five experience clinically significant interpersonal problems (Author, work in preparation), highlighting the importance of supporting mental health carers in their interpersonal functioning.

Interpersonal problems are difficulties encountered when interacting with others, and represent a common reason why individuals seek psychotherapy (Horowitz, Rosenberg, \& Bartholomew, 1993). Support for mental health carers' to address relationship difficulties is targeted through a range of interventions such as group interventions for expressed emotion (Sadath, Muralidhar, Varambally, \& Gangadhar, 2017), mindfulness for empathic perspective taking (Birnie, Speca, \& Carlson, 2010) and communication tools (Done \& Thomas, 2001; Young, Manthorp, Howells, \& Tullo, 2011). However, such interventions target specific relational difficulties in isolation rather than interpersonal functioning as a whole. In addition, 
the most common outcome measures utilised in mental health carer interventions are psychological distress and carer burden (Arksey, 2003; Yesufu-Udechuku et al., 2015). To the author's knowledge, there is no current research utilising interpersonal problems as an outcome of mental health carer intervention, despite the high prevalence.

Interventions targeting interpersonal functioning need to address the psychological processes thought to maintain relational dysfunction (Alden \& Capreol, 1993). Several theories of interpersonal problems point to the role of experiential avoidance in contributing to or perpetuating relational dysfunction (Holtforth, 2008; Holtforth, Bents, Mauler, \& Grawe, 2006; Inge, 1992; Sullivan, 1953). Experiential avoidance is defined as attempts to avoid internal stimuli (e.g., cognitions, emotions, memories) even when doing so creates harm (Hayes, Strosahl, \& Wilson, 1999). Experiential avoidance has been associated with a host of negative outcomes (Chawla \& Ostafin, 2007; Cristea, Montgomery, Szamoskozi, \& David, 2013; Hayes, Luoma, Bond, Masuda, \& Lillis, 2006; Lillis, Levin, \& Hayes, 2011) and caregivers have been found to exhibit moderate to high levels of experiential avoidance (Ulstein, Wyller, \& Engedal, 2008). Recent empirical studies indicate a strong relationship between experiential avoidance and interpersonal problems in the general population (Gerhart, Baker, Hoerger, \& Ronan, 2014) and mental health caregiver populations (reference removed for blind review). Moreover, experiential avoidance predicts negative expectations of relationships in mental health carers (reference removed for blind review). Negative expectations of relationships are strongly held beliefs about self and others that contribute to rigid patterns of interpersonal behaviour (Downey, Freitas, Michaelis, \& Khouri, 1998). In turn, these expectations have been found to mediate the relationship between experiential avoidance and particular types of interpersonal problems in mental health carer's (identifying reference). It follows that an intervention designed to reduce mental health carer's interpersonal problems would target experiential avoidance and negative expectations of 
relationships.

Acceptance and Commitment Therapy (ACT) was designed explicitly to target experiential avoidance (Hayes et al., 1999) and thus has featured prominently in the experiential avoidance literature (Chawla \& Ostafin, 2007). Acceptance and Commitment Therapy (ACT) is a 'third wave' behaviour therapy intervention that aims to improve quality of life by addressing ineffective control strategies and promoting value directed behaviour change (Hayes et al., 2006). ACT represents a promising intervention for caregiver populations in a range of contexts. Reported benefits include reductions in psychological distress and caregiver burden, found in dementia carers (Franco, Sola, \& Justo, 2010); reductions in depression and general distress, found in parents of children with Autism (Blackledge \& Hayes, 2006); reductions in parenting distress, found in parents of children with intellectual disabilities (Rayan \& Ahmad, 2017); and reductions in psychological distress, found in support staff (Noone \& Hastings, 2011).

ACT targets experiential avoidance by promoting acceptance- defined as 'actively contacting psychological experiences - directly, fully, and without needless defence- while behaving effectively’ (Hayes, Wilson, Gifford, Follette, \& Strosahl, 1996, p.1163). Acceptance is associated with a range of positive outcomes (for a review see Williams \& Lynn, 2010) and is related to emotional wellbeing in caregivers (Beer, Ward, \& Moar, 2013). Substantial evidence indicates that ACT decreases experiential avoidance in both clinical and normal populations (see Choi, Vickers, \& Tassone, 2014 for a review) and increases willingness to engage in activities whilst experiencing difficult emotions (Eifert \& Heffner, 2003; Levitt, Brown, Orsillo, \& Barlow, 2004). In relation to carers, a study comparing the impact of ACT and CBT found that whilst both reduced depressive symptoms in carers, only the former was associated with reductions in caregiver's experiential avoidance (Losada et al., 2015). A growing body of research argues that targeting experiential avoidance using an 
acceptance framework is particularly applicable to the carer population, as the realities of mental health caregiving are not always amendable to the problem solving techniques of CBT (Losada et al., 2015; Leoni, Corti, Cavagnola, Healy, \& Noone, 2016). The significance of this is further highlighted by some research that has found caregivers' attempts to directly reduce or solve unpleasant emotional experiences is associated with increased stress and burden (Devereux, Hastings, \& Noone, 2009).

Mindfulness- a key element of ACT interventions- is described as a state of being open and aware (Hayes, 2004). Mindfulness has been associated with reductions in interpersonal problems (Millstein, Orsillo, Hayes-Skelton, \& Roemer, 2015), increased emotional regulation and decreased reactivity (Davis \& Hayes, 2011), increased social connectedness (Cohen \& Miller, 2009) and improved relationship functioning (Carson, Carson, Gil, \& Baucom, 2004). Mindfulness has the potential to reduce negative expectations of relationships, in particular the subskill of cognitive defusion. Negative, excessive and/or unrealistic expectations of relationships can evolve when individuals hold inflexible rules about how relationships are "supposed" to be. They also involve strong judgements about people and their interactions with them (e.g., you are inconsiderate). Cognitive fusion has been described as a process where people get stuck to such thoughts and have difficulty separating themselves from these thoughts to the extent that they come to dominate their behaviour. Cognitive defusion describes the process of perceiving thoughts as mental events rather than literal truths (Hayes et al., 2006) and assists one to make behavioural choices based on values rather than falling into automatic patterns. For example, cognitive defusion may assist a mental health carer to recognise their negative expectation "What's the point, they won't listen" as just a thought instead of responding as though it and the meaning behind it (e.g., he/she does not respect or care for me) are truths. Cognitive defusion is thought to facilitate the capacity of people to not respond as though their beliefs were factual and in this 
instance to work towards their value of communication even in the face of hostile or anxious feelings. Studies in this area suggest that cognitive defusion is associated with less believability and emotional impact of negative thoughts (Mandavia et al., 2015; Masuda et al., 2010), and predicts more approach and less avoidance coping behaviour (Donald, Atkins, Parker, Guo, \& Christie, 2017).

As outlined, ACT has a strong theoretical foundation for addressing interpersonal problems in mental health carers. However, carer interventions with two or more conceptually different approaches have achieved more positive outcomes (Carers NSW, 2017; Dickinson et al., 2016; Laver, Milte, Dyer, \& Crotty, 2016). For example, the Resources for Enhancing Caregiver Health (REACH) project found positive benefits for a multicomponent carer intervention that combined stress management, links to community support, and caregiver training (Elliott, Burgio, \& DeCoster, 2010). Additionally, multicomponent interventions have been found to be most effective when delivered in a group format which allows for peer support and networking (Dickinson et al., 2016; Carers NSW, 2017). As multicomponent interventions are recognised as best practice for carer populations (Dickinson et al., 2016; Carers NSW, 2017), this suggests the utility of an additional approach alongside ACT when addressing carer's interpersonal problems. McKay, Lev and Skeen (2012) presented a theoretical framework in which ACT concepts can be delivered within a schema based formulation in order to address interpersonal problems. A protocol for an ACT and Schema group intervention has been found to be effective at helping individuals overcome maladaptive interpersonal behaviour (Lev, 2011; McKay et al., 2012). Although not commonly used within the caregiving field, schema therapy was designed to assist individuals to change maladaptive patterns in thought and behaviors (Beck, 1964). Central to this approach is the concept of 'schemas', defined as longstanding cognitive frameworks regarding self and others (Beck, 1964; Young, Klosko, \& Weishaar, 2006). 
Schemas have a strong history within the interpersonal development field and have been posed to contribute to and maintain interpersonal dysfunction (Beckley, 2011; Douglas, Binder, Kajos, Hyde, \& Li, 2013; Thimm, 2013). Schemas derived from attachment styles have been found to be linked to parents' negative attributions of their children's behaviours (e.g., Grusec, Hastings, \& Mammone, 1994). Schema therapy aims to identify and alter these frameworks, and may for example, assist a mental health carer to recognize their negative expectation "What's the point, they never listen" is a result of past experiences of emotional deprivation and not a reflection of present truths. The use of schema interventions with the caregiver population is rare, with only one study incorporating this in therapy with carers of those entering hospice (Lindstrom \& Melnyk, 2013). Results indicated reduced anxiety, increased preparedness for the role, increased confidence, and increases in helpful beliefs in carers who engaged in the schema intervention (Lindstrom \& Melnyk, 2013).

The use of an ACT and Schema group intervention with the mental health carer population has a strong justification: 1) There is a theoretical rationale for the role of experiential avoidance and negative expectations of relationships (and associated cognitive fusion) in underpinning mental health carers interpersonal problems; 2) Correlational data demonstrates that the processes targeted by ACT (i.e. experiential avoidance) and Schema therapy (i.e. negative expectations of relationships) are associated with mental health carer's experiences of interpersonal problems; 3) Existing studies demonstrate positive results for ACT based interventions with caregiver populations; 4) Multi-component carer interventions with two conceptually different approaches have achieved more positive outcomes in prior studies. The current study aims to pilot an ACT and Schema group intervention for mental health carers' interpersonal problems, examining acceptability and conducting preliminary assessment of effectiveness. 


\section{Method}

\section{Study design}

This pilot study had a mixed methods design, incorporating both quantitative and qualitative data. Quantitative data was gained through assessment booklets administered at week one, week six and week twelve of the program, with two additional measures administered on a weekly basis. Qualitative data was gained through focus groups conducted three months post intervention. This study was approved by the University of Wollongong Human Research Ethics Committee.

\section{Pilot testing and participants}

The program was pilot tested in three community mental health organisations in the state of New South Wales, Australia. Due to the length of the program and constraints of service capacity, multiple programs were conducted over the period of late 2015 to mid-2017. On each occasion, the service advertised the program to their current client base using flyers and word of mouth. Interested caregivers were invited to attend an intake meeting where suitability for the program was assessed and information on the research provided. Exclusion criteria included 1) acute crisis, 2) moderate to high suicide risk, 3) mental health or cognitive difficulties that would significantly impact upon engagement, 4) lack of insight into interpersonal difficulties, 5) inability to commit to the twelve week program. Caregivers provided written consent at the intake meeting.

\section{Intervention}

The intervention consisted of a group program aimed to assist mental health caregivers to build stronger and more mindful relationships, titled 'Me and My Relationships' (MMR). The program was closely based on an existing 10 week protocol by McKay et al. (2012). McKay 
et al.'s (2012) protocol utilises schemas as a functional theme, introducing common schema patterns that are relevant to interpersonal problems (e.g. abdonment, entitlement, subjugation), which are addressed using ACT methods (e.g. developing mindfulness skills, clarifying values, cognitive defusion). For the purposes of the current study, adaptations included the expansion of early psychoeducation on schemas, utilisation of vignettes specific to the caregiving role, and the introduction of an eco-map (diagram of social and personal relationships). The MMR program was structured over 12 weeks, with each session of 1.5 hours duration. Program content is outlined in Table 1. A detailed facilitator's handbook was developed in order to ensure consistency in delivery across sites and a client handbook containing information and worksheets was provided to participants. The main facilitator held a Bachelor's degree in psychology and provisional registration as a psychologist. Cofacilitation was by a nominated staff member of the host organisation, who was required to hold bachelor level qualifications in psychology, counselling or social work. Training consisted of a one-day ACT workshop and weekly supervision. To ensure consistency in delivery, weekly supervision occurred for the duration of the program, provided by the first author who is a psychologist and accredited supervisor through the Psychology Board of Australia.

\section{Measures}

In addition to information on socio-demographic data, several self-report questionnaires were utilised. All measures were collected at commencement of the program (week one), at the mid-point (week six) and at the final week (week twelve). In addition, two measures (the Outcome Rating Scale and Session Rating Scale) were completed on a weekly basis.

\section{Interpersonal problems}


Interpersonal problems were measured utilising the Inventory of Interpersonal Problems 32 (IIP-32: Barkham, Hardy, \& Startup, 1996; Horowitz, Rosenberg, Baer, Ureno, \& Villasenor, 1988). The IIP-32 examines eight categories of interpersonal problems: domineering/controlling, vindictive/self-centred, cold/distant, socially inhibited, nonassertive, overly accommodating, self-sacrificing and intrusive/needy (Horowitz, Alden, Wiggins, \& Pincus, 2000). Containing 32 items, it produces eight subscales that correspond to these eight categories. Example items include 'I find it hard to really care about other people's problems' (vindictive/self-centred) and 'I let other people take advantage of me too much' (overly accommodating). Questions are answered on a 5-point Likert scale $(0=$ not at all, 4 = extremely), summed and standardized according to community norms. A T-score of 50 represents the mean, with a score of 60 or greater indicating above average difficulty, and a score of 70 or greater indicating significant difficulty (Horowitz et al., 2000). The IIP has high internal and test-retest reliability and convergent and criterion validity (Alden, Wiggins, \& Pincus, 1990; Barkham et al., 1996; Horowitz et al., 1988).

\section{Experiential avoidance}

Experiential avoidance of painful emotions and uncomfortable situations was measured using the Brief Experiential Avoidance Questionnaire (BEAQ: Gámez et al., 2014). It consists of 15 items (e.g. 'I work hard to keep out upsetting feelings') measured on a 6point Likert scale ( 1 = strongly disagree, $6=$ strongly agree). Responses are summed with higher values indicating greater experiential avoidance. The BEAQ contains Cronbach's alphas ranging from .80 to .89 (Gámez et al., 2014).

\section{Caregiving avoidance}

The avoidance of emotions, thoughts and sensations specific to caregiving was measured using the Experiential Avoidance in Caregiving Questionnaire (EACQ: Losada, 
Márquez-González, Romero-Moreno, \& López, 2014) . The EACQ contains 15 items (e.g., 'thinking too much about what a caregiver feels and thinks about his/her caregiving situation is harmful') answered on a 5-point Likert scale ( $1=$ 'not at all', $6=$ 'a lot'). Responses are summed with higher values associated with greater experiential avoidance within the caregiving context. The EACQ shows acceptable psychometric properties with a Cronbach's alpha of 0.70 (Losada et al., 2015).

\section{Mindfulness}

Dispositional mindfulness (i.e., inherent capacity) was measured using the Mindfulness Awareness Attention Scale (MAAS: Brown \& Ryan, 2003). It consists of 15 items (e.g., 'I find myself doing things without paying attention') answered on a 6-point

Likert scale ( 1 = 'almost always', 6 = 'almost never'). Items are summed, with higher scores indicating higher levels of dispositional mindfulness. The MAAS-15 has been shown to be a reliable and valid instrument for use in general adult populations, with a Cronbach's alpha ranging from 0.82 to 0.87 (Brown \& Ryan, 2003).

\section{Wellbeing}

Wellbeing was measured using the Outcome Rating Scale (ORS: Miller, Duncan, Brown, Sparks, \& Claud, 2003). The ORS is a visual analogue scale with four items that measure individual well-being, interpersonal well-being, social well-being and general wellbeing. Participants rate how they feel that that area of their life has been for them over the course of a week on a line ranging from 0 to 10 . Items are scored and totalled using a $10 \mathrm{~mm}$ ruler. Scores of approximately 25 representing optimal wellbeing with a ceiling effect of 40 . This measure has a reported coefficient alpha of .93 and good internal consistency and testretest reliability (Miller et al., 2003; Bringhurst, Watson, Miller, \& Duncan, 2006). 


\section{Acceptability of the intervention}

In addition to retention and participation rates, participant's perception of the usefulness and effectiveness of the MMR program was assessed using the Session Rating Scale (SRS: Duncan et al., 2003). The SRS is a visual analogue scale that provides feedback on four items: perceptions of the therapeutic relationship, goals and topics, approach and methods, and overall satisfaction. Participants indicate how well they feel the delivered intervention meets each criterion on a line ranging from 0 to 10 . Items are scored and totalled using a $10 \mathrm{~mm}$ ruler. Higher scores indicate greater satisfaction with the intervention, with optimal scores in the 36-40 range. The SRS demonstrates impressive internal consistency and test-retest reliability (Miller et al., 2003; Duncan et al., 2003).

\section{Focus groups}

Three months following the first MMR group, participants were invited to attend a focus group to explore their experience of the program and its impact on their caring relationships. This process was repeated for the second and third group. Focus group participants were provided with an information sheet and written consent was obtained. Each focus group was facilitated by the first author, who was not directly involved in delivering the intervention. Questions included: 1) Has the program assisted you with managing your interpersonal difficulties with the person you are caring for? (If yes: how?), 2) What changes (if any) have you noticed in your relationships since completing the program? 3) Since completing the program, have you noticed any change in your capacity to accept or your tendency to avoid difficult relationship experiences? (If yes: please describe these changes),

4) Could you describe any helpful events during the program? 5) Could you describe any hindering events during the program?

\section{Data analysis}




\section{Quantitative}

A series of one-way repeated measure ANOVAs were conducted to assess changes on all outcome measures over the three time points (Weeks 1, 6, 12). Normality tests indicated our variables of caregiving avoidance, experiential avoidance, mindfulness and wellbeing were all normally distributed, with mild to moderate skewness present for several of the interpersonal problem domain variables. Where variables displayed skewness, nonparametric equivalents were conducted. Mauchly's test of sphericity was assessed for all ANOVAs, with a Greenhouse Geisser correction applied where this assumption was not met. Where ANOVAs indicated significant effects by time, we conducted stepdown paired comparisons using a Bonferroni correction to control for Type 1 errors. Cohen's $d$ effect sizes for significant paired comparisons were calculated using http://www.socscistatistics.com/effectsize/Default3.aspx (accessed 17 October 2017). Interpretation was as follows: 0.2 to 0.4 represents a small effect, 0.5 to 0.7 represents a medium effect, 0.8 and above represents a large effect (Cohen, 1988). An intent to treat analysis using multiple imputation for missing data was also conducted. All analyses were conducted using the IBM SPSS Statistics package version 21.

In light of suggestions that measuring only the mean interpersonal problem value on the IPP-32 negates individual differences (Salzer, Winkelbach, Leibing, Pincus, \& Leichsenring, 2011), additional analysis were undertaken. We examined reductions in problematic interpersonal problems on an individual level and noted which participants saw reductions from the above average range to the average range. To assess acceptability, we examined mean scores on the SRS, number of sessions attended by each individual and the timing of missed sessions, and we noted whether any participants dropped out of the program and at what point this occurred. 


\section{Qualitative}

Qualitative analysis consisted of thematic analysis guided by the steps outlined by Braun and Clarke (2006). Focus group dialogues were transcribed verbatim and de-identified labels were used in the interest of confidentiality. Initial codes and themes were developed using a grounded theory approach following careful reading and re-reading of transcripts by two researchers. To assist with interrater reliability, a manual was created which listed codes, descriptions of each code, example quotes and emergent themes. Codes and themes underwent successive rounds of comparison, within and across focus groups, as we compared their content and meaning in relation to one another and to the dataset in its entirety. When discrepancies were present, themes and definitions were reviewed and discussed against the raw data set until agreement was met. Once the list of themes was finalised, a name was given to each theme thought to capture its essence and the final report was produced.

\section{Results}

As shown in the study flowchart (Figure 1), 34 participants attended an intake meeting. Two decided that they did not wish to take part in the program, and four were deemed not appropriate according to exclusion criteria. Thus 28 clients began the program, of which 24 successfully completed. All participants were caring for a family member; which consisted of parents $(50 \%)$, spouse $(29.2 \%)$, siblings $(8.3 \%)$ and other relatives $(12.5 \%)$. The vast majority of participants were women (83.3\%). Just over half (58.3\%) of participants were long term carers, having cared for the care receivers for over 10 years. The mental health condition of the care-receiver's were identified by the carer, with depression $(41.7 \%)$ and anxiety $(54.2 \%)$ the most frequently reported. More than one condition could be identified for each care recipient and other conditions included Bipolar Disorder (25\%), Post-Traumatic

Stress Disorder (20.8\%), Borderline Personality Disorder (29.2\%), Schizophrenia (20.8\%) 
and Substance Use Difficulties (8.3\%). The majority of carers (45.8\%) identified they perceived the care-receiver to be in recovery, with the remaining participants disagreeing $(25 \%)$ or reporting they were unsure $(29.2 \%)$. Of this original sample, $50 \%(n=12)$ took part in focus groups.

\section{Acceptability}

Retention and participation rates were high. The vast majority of participants (85\%) who commenced the program completed it. Of the four participants who withdrew, all did so during the first half of the program (week 2; week 2; week 3 and week 5). Attendance for participants who completed the program was consistently high, with 11 participants $(46 \%$ of sample) attending the entire program, 11 participants (46\%) attending 11 of the 12 sessions, and 2 participants ( $8 \%$ ) attending 10 of 12 sessions. Across the entire pilot, the average number of sessions attended was 11.38 for completed participants, and 10.18 when including the four participants who withdrew from the program. The vast majority (80\%) of missed sessions occurred in the second half of the program, with weeks 8 to 10 a period of risk that accounted for $60 \%$ of the total missed sessions.

The Session Rating Scale indicated consistently positive feedback on the program, with an average score of 35.8 at week one $(n=24), 36.5$ at week six $(n=24)$ and 38.7 at week twelve $(n=24)$. The average SRS score across all weeks and all five groups was 37.3 out of a possible $40(n=273)$.

\section{Preliminary testing of effectiveness}

Descriptive statistics and repeated measure ANOVA results are presented in Table 2. Ten of the thirteen ANOVAs indicated that there were significant differences on measures over time. Stepdown paired comparisons indicated total interpersonal problems significantly reduced between week one and twelve (Mdiff $=8.55,95 \%$ CI 2.28 to 14.80$)$ with a large effect size $(d$ 
$=0.86$ ). On the domain level, domineering/controlling significantly reduced between week six and twelve (Mdiff $=3.46,95 \%$ CI 2.26 to 6.66$)$ with a small effect size $(d=0.39)$.

Cold/distant reduced between week one and twelve $($ Mdiff $=6.67,95 \%$ CI .14 to 13.19$)$ with a medium effect size $(d=0.55)$. Non-assertive reduced between week one and twelve (Mdiff $=8.29,95 \%$ CI 1.42 to 15.16$)$ with a medium effect size $(d=0.70)$. Overly accommodating reduced between week one and $\operatorname{six}(\operatorname{Mdiff}=6.84,95 \%$ CI 1.02 to 12.64$)$ with a medium effect size $(d=0.58)$, in addition to week one and twelve (Mdiff $=8.38,95 \%$ CI 1.97 to 14.78), medium effect size $(d=0.73)$. Due to the presence of mild to moderate skewness on several interpersonal problem variables, nonparametric Friedman's tests were conducted with Wilcoxon Signed Ranks tests to assess paired comparisons. All nonparametric tests were consistent with those from the ANOVA results with regard to significant effects.

On our remaining variables, experiential avoidance significantly reduced between week one and twelve (Mdiff $=10.04,95 \%$ CI 4.30 to 15.78$)$ with a large effect size $(d=$ 0.89). Caregiving avoidance reduced between week six and twelve (Mdiff $=5.13,95 \% \mathrm{CI}$ 1.72 to 8.53$)$ with a medium effect size $(d=0.73)$, in addition to week one and twelve (Mdiff $=7.63,95 \%$ CI 3.92 to 11.33$)$, large effect size $(d=1.04)$. Mindfulness increased between week one and six (Mdiff $=-7.46,95 \%$ CI -12.46 to -2.45$)$ with a medium effect size $(d=$ 0.59), in addition to week one and twelve (Mdiff $=-10.13,95 \%$ CI -15.89 to -4.36 ), large effect size $(d=0.84)$. Wellbeing increased between week one and six (Mdiff $=-5.21,95 \% \mathrm{CI}$ -9.76 to -.66$)$ with a medium effect size $(d=0.63)$, week six and twelve (Mdiff $=-5.6,95 \%$ CI -9.46 to -1.75$)$ with a medium effect size $(d=0.72)$ and week one and twelve (Mdiff $=$ $10.81,95 \%$ CI -14.46 to -7.16$)$ with a large effect size $(d=1.50)$.

We also examined the number of individuals scoring in the above average range $(\geq 60)$ for interpersonal problem domains across the three time points of the intervention. As indicated in Table 3, the total interpersonal problem score displayed a $71.4 \%$ decrease 
between week one and twelve. At the domain level the number of participants scoring in the above average range reduced by at least $50 \%$, with a range of $50 \%$ (domineering/controlling) to $62.5 \%$ (self-sacrificing).

\section{Intention-to-treat analysis}

Although the study was predominantly interested in the outcomes of those who completed the program an intention-to-treat analysis was also conducted to assess whether attrition (4 participants) from the program might have affected the findings. Multiple imputation (MI) for missing data was used. Missingness was based on absence of participants (attrition) particularly at follow-up assessments. Five demographic variables (carer gender, relationship to care recipient, time caring, intervention group, and whether carer felt care receiver was in recovery) and all 13 dependent variables (all measures at all time points) were used to impute missing values. Five imputations were conducted. The results from all repeated measures ANOVAs replicated the pattern of findings in the "per protocol" analysis reported in Table 3 for 12 of the 13 dependent variables. The one exception was the self-sacrificing interpersonal problem domain- which was significant at the 0.05 level on the "per protocol" analysis, however displayed significance on only four of the five imputations.

\section{Qualitative results}

Focus groups were held with prior participants of group one $(n=4)$, group two $(n=4)$ and group three $(n=4)$. All twelve participants attended, representing $50 \%$ of the original sample. Focus groups ran for approximately one hour duration and contained set questions regarding changes in relationships and acceptance since completing the program, in addition to discussion of helpful and hindering events. The results of the thematic analysis are presented in Table 4. 
As can be seen in Table 4, participants discussed aspects of the MMR program and described positive changes in emotions such as reduced emotion frequency and intensity. They reported reduced reactivity, increased acceptance of their caregiving situation, improvements in communication, changes in agency and increased connection with the care recipient either during the MMR program and/or in the three months following program completion. Of the twelve participants involved in the focus groups, three identified hindering events such as initial confusion with concepts, external factors influencing attendance, and feeling that the sessions went by too quick. However, the low frequency of these hindering events was not sufficient to constitute a theme.

\section{Discussion}

This study aimed to explore whether an ACT and Schema group program would be acceptable to a mental health carer population and show preliminary effectiveness. Findings indicate that the interpersonal problem domains of domineering/controlling, cold/distant, non-assertive, overly accommodating, self-sacrificing- as well as the total interpersonal problem score- significantly reduced, when comparing measures collected at the last week of the program with those collected at the first week. This result is consistent with literature indicating interpersonal problems pertaining to overly accommodating and self-sacrificing experience the most gains from psychotherapy (Cain, Pincus, \& Holtforth, 2010; Horowitz et al., 1993; Renner et al., 2012). The interpersonal problem domains of vindictive/self-centred, socially inhibited and intrusive needy were not associated with statistically significant change over time. Two of these domains- vindictive/self-centred and intrusive/needy- have been identified as least amendable to psychotherapy (Cain et al., 2010; Horowitz et al., 1993; Renner et al., 2012). However, as participants commenced with different interpersonal profiles, group results should be interpreted with caution as individual changes can be masked. For example, excessively non-assertive carers may have increased in dominance and 
excessively domineering carers may have increased in non-assertiveness. The number of participants presenting with above average interpersonal problems reduced over time by at least $50 \%$ for each domain, with our three non-significant domains reducing by $66 \%$ (vindictive/self-centred), 50\% (socially inhibited) and 58\% (intrusive/needy). Qualitative findings provide insight into potential processes for managing interpersonal problems, with participants noting the importance of connection, communication, agency and processing of emotions.

Benefits outside of interpersonal functioning were also evident, with findings indicating participant's perceived mindfulness and wellbeing significantly increased, and perceived experiential and caregiving avoidance significant decreased, at the last week of program compared to the first. This is consistent with a large body of research on the impact of ACT-based interventions for caregiver populations (Leoni et al., 2016; Losada et al., 2015; Noone \& Hastings, 2010; McConachie, McKenzie, Morris, \& Walley, 2014). Qualitative results provided some insight into these changes, with participants noting increased emotional regulation, decreased reactivity, increased response flexibility and improved relationshipsconsistent with what we know to be the benefits of mindfulness (Davis \& Hayes, 2011). In addition, participants noted increased willingness and acceptance- consistent with the experiential avoidance literature (Hayes et al., 1996). Although this study was not designed to determine causal relationships between variables, previous literature suggests that experiential avoidance may be a mechanism of change in relation to interpersonal functioning. Considering experiential avoidance has been found to mediate the effect of mindfulness on multiple outcomes (for a review, see Weinrib, 2011), further investigation of the relationships between these variables is required.

Focus group responses, SRS scores, attendance and retention rates indicated that the MMR program was highly acceptable and no major modifications to the content are 
anticipated. Findings highlight the importance of retaining participants in the first half of the program, and continuing to encourage attendance in the second half of the program, particularly during the high risk period of week 8 to 10 . Content during this period included structured goal setting and review, which may have generated internal discomfort and avoidant behaviour for participants. Future programs may benefit from emphasising the concept of committed action- acting in accordance with one's values despite the experience of discomfort- during this time. Finding that the majority of significant change in the current study occurred between week one and week twelve suggests the full duration of the program is needed for maximum benefit. This is somewhat surprising in light of research suggesting the effect of therapy is greatest in early sessions with less rapid rates of change seen over time (Kopta, 2003; Stulz, Lutz, Kopta, Minami, \& Saunders, 2013). The necessity of all twelve weeks may be explained by the sequential skill building structure of the MMR program, which contains an early emphasis on conceptual based exposure and gradual move towards behavioural implementation of core skills. Additionally, entrenched interpersonal problem patterns may require more time to alter, especially if these are supported by wellestablished schema. Although the full program is relatively intensive, the success of the current pilot across three community organisations suggests promise for its future feasibility.

Considering the difficulties caregivers face in obtaining support due to time, distance and competing demands (Bormann et al., 2009; Moore \& McArthur, 2007), the accessibility of the MMR program needs to be considered. On average, only 4.8 carers attended each program; the intervention was designed to accommodate approximately 8 caregivers. Our study utilised participants who self-identified as experiencing interpersonal difficulties, however the use of a standardized screening process may assist in identifying additional carers who may benefit from the program. The Inventory of Interpersonal Problems- which provides a direct connection between assessment, interpersonal theory and intervention- 
represents one avenue for achieving this (Alden et al., 1990; Horowitz et al., 2000). Flexible modes of intervention delivery, such as correspondence (Deane, Marshall, Crowe, White, \& Kavanagh, 2015) or technology assisted approaches (Scott et al., 2016) have shown promise for the caregiver population, and represent an additional avenue for improving the access of future MMR programs.

This study has several limitations which should be noted. The sample size was small and its self-selected nature means it is not representative of all mental health carers or their interpersonal profiles. The design of the study- open pilot with the absence of control groupdoes not allow for causal conclusions about the impact of the intervention. There were also a number of limitations in regards to our outcome measures. First, our measures were primarily ACT based, with no measures to assess schema. The inclusion of schema outcome measures may have assisted in capturing underlying schema modes that are related to negative expectations of relationships. Second, our measures were self-report in nature and relied exclusively on the mental health carers' perception of their relationships. Considering carers and care-receivers experience disparity in how they view their relationship (Manne et al., 2006), this study could have been improved by incorporating care-receivers perceptions of interpersonal functioning. Third, the collection of final measures at the last week of programchosen to maximise completion rates given the small sample size- limits the generalizability of outcomes over time. Although post-intervention focus groups provided some indication of sustainability of change, the addition of longitudinal quantitative data would have enhanced our study.

\section{Conclusion}

Although tentative, findings provide preliminary support for the utility of an ACT and Schema group intervention for improving interpersonal functioning in mental health carers. 
Given the high prevalence of interpersonal problems in this population (Author, work in preparation) and paucity of research in the area, further investigation is needed. Ideally, this would take the form of an adequately powered randomized controlled trial (RCT). The RCT could determine potential benefits of the intervention as compared to a control group, incorporate schema outcome measures and longitudinal data in the study design, and ascertain accessibility of the program. 


\section{References}

Alden, L., \& Capreol, M. (1993). Avoidant personality disorder: Interpersonal problems as predictors of treatment response. Behavior Therapy, 24(3), 357-376, doi:10.1016/S0005-7894(05)80211-4.

Alden, L., Wiggins, J., \& Pincus, A. (1990). Construction of circumplex scales for the Inventory of Interpersonal Problems. Journal of Personality Assessment, 55(3-4), 521-536. doi:10.1207/s15327752jpa5503\&4_10

Arksey, H. (2003). Scoping the field: services for carers of people with mental health problems. Health \& Social Care in the Community, 11(4), 335-344, doi:

10.1046/j.1365-2524.2003.00433.x

Barkham, M., Hardy, G. E., \& Startup, M. (1996). The IIP-32: A short version of the Inventory of Interpersonal Problems. British Journal of Clinical Psychology, 35(1), 21-35, doi:10.1111/j.2044-8260.1996.tb01159.x.

Beck, A. T. (1964). Thinking and depression. II. Theory and therapy. Archives Of General Psychiatry, 10, 561-571, doi:10.1001/archpsyc.1964.01720240015003.

Beckley, K. (2011). Making Sense of Interpersonal Dynamics: A Schema Focused Approach. In P. G. Willmot, N. (Ed.), Working Positively with Personality Disorder in Secure Settings: A Practitioner's Perspective (pp. 172-187): John Wiley \& Sons.

Beer, M., Ward, L., \& Moar, K. (2013). The relationship between mindful parenting and distress in parents of children with an autism spectrum disorder. Mindfulness, 4(2), 102-112, doi:10.1007/s12671-012-0192-4.

Birnie, K., Speca, M., \& Carlson, L. E. (2010). Exploring self-compassion and empathy in the context of mindfulness-based stress reduction (MBSR). Stress \& Health: Journal of the International Society for the Investigation of Stress, 26(5), 359-371, doi:10.1002/smi.1305.

Blackledge, J. T., \& Hayes, S. C. (2006). Using Acceptance and Commitment Training in the Support of Parents of Children Diagnosed with Autism. Child \& Family Behavior Therapy, 28(1), 1-18, doi:10.1300/J019v28n01_01.

Bormann, J., Warren, K. A., Regalbuto, L., Glaser, D., Kelly, A., Schnack, J., \& Hinton, L. (2009). A spiritually based caregiver intervention with telephone delivery for family caregivers of veterans with dementia. Family \& Community Health, 32(4), 345-353, doi:10.1097/FCH.0b013e3181b91fd6. 
Braithwaite, V. (2000). Contextual or general stress outcomes: Making choices through caregiving appraisals. The Gerontologist, 40(6), 706-717, doi:10.1093/geront/40.6.706.

Braun, V., \& Clarke, V. (2006). Using thematic analysis in psychology. Qualitative Research in Psychology, 3(2), 77-101, doi:10.1191/1478088706qp063oa.

Bringhurst, D. L., Watson, C. W., Miller, S. D., \& Duncan, B. L. (2006). The reliability and validity of the Outcome Rating Scale: a replication study of a brief clinical measure. Journal of Brief Therapy, 5, 23-30.

Brown, K. W., \& Ryan, R. M. (2003). The benefits of being present: Mindfulness and its role in psychological well-being. Journal of Personality and Social Psychology, 84 (4), 822-848, doi:10.1037/0022-3514.84.4.822.

Cain, N. M., Pincus, A. L., \& Holtforth, M. G. (2010). Interpersonal Subtypes in Social Phobia: Diagnostic and Treatment Implications. Journal of Personality Assessment, 92(6), 514-527, doi:10.1080/00223891.2010.513704.

Carers NSW. (2017). Carer Counselling Literature Review.

Carers Recognition Act NSW. (2010). Available from https://www.legislation.nsw.gov.au/\#/view/act/2010/20/whole.

Carson, J. W., Carson, K. M., Gil, K. M., \& Baucom, D. H. (2004). Mindfulness-based relationship enhancement. Behavior Therapy, 35(3), 471-494, doi:10.1016/S00057894(04)80028-5.

Chattoo, S., \& Ahmad, W. I. U. (2008). The moral economy of selfhood and caring: Negotiating boundaries of personal care as embodied moral practice. Sociology of Health and Illness, 30(4), 550-564, doi:10.1111/j.1467-9566.2007.01072.x.

Chawla, N., \& Ostafin, B. (2007). Experiential avoidance as a functional dimensional approach to psychopathology: An empirical review. Journal of Clinical Psychology, 63(9), 871-890, doi:10.1002/jclp.20400.

Choi, K., Vickers, K., \& Tassone, A. (2014). Trait Emotional Intelligence, Anxiety Sensitivity, and Experiential Avoidance in Stress Reactivity and Their Improvement Through Psychological Methods. Europe's Journal of Psychology, 10(2), 376-404, doi:10.5964/ejop.v10i2.754.

Cohen, J. (1988). Statistical Power Analysis for the Behavioral Sciences. Hillsdale: Lawrence Erlbaum. 
Cohen, J., \& Miller, L. (2009). Interpersonal mindfulness training for well-being: A pilot study with psychology graduate students. Teachers College Record, 111(12), 27602774.

Cristea, I. A., Montgomery, G. H., Szamoskozi, Ş., \& David, D. (2013). Key Constructs in "Classical" and "New Wave" Cognitive Behavioral Psychotherapies: Relationships Among Each Other and With Emotional Distress. Journal of Clinical Psychology, 69(6), 584-599, doi:10.1002/jclp.21976.

Davis, D., \& Hayes, J. (2011). What are the benefits of mindfulness? A practice review of psychotherapy-related research. Psychotherapy, 48(2), 198-208, doi:10.1037/a0022062.

Deane, F. P., Marshall, S., Crowe, T., White, A., \& Kavanagh, D. (2015). A Randomized Controlled Trial of a Correspondence-Based Intervention for Carers of Relatives with Psychosis. Clinical Psychology \& Psychotherapy, 22(2), 142-152, doi:10.1002/cpp.1880.

Devereux, J., Hastings, R., \& Noone, S. (2009). Staff stress and burnout in intellectual disability services: Work stress theory and its application. Journal of Applied Research in Intellectual Disabilities, 22(6), 561-573, doi:10.1111/j.14683148.2009.00509.x.

Dickinson, C., Dow, J., Gibson, G., Hayes, L., Robalino, S., \& Robinson, L. (2016). Psychosocial intervention for carers of people with dementia: What components are most effective and when? A systematic review of systematic reviews. International Psychogeriatrics, 29(1), 31-43, doi:10.1017/S1041610216001447.

Donald, J. N., Atkins, P. W. B., Parker, P. D., Guo, J., \& Christie, A. M. (2017). Cognitive Defusion Predicts More Approach and Less Avoidance Coping With Stress, Independent of Threat and Self-Efficacy Appraisals. Journal of Personality, 85(5), 716-729, doi:10.1111/jopy.12279.

Done, D. J., \& Thomas, J. A. (2001). Training in communication skills for informal carers of people suffering from dementia: a cluster randomized clinical trial comparing a therapist led workshop and a booklet. International Journal of Geriatric Psychiatry, 16(8), 816-821, doi: 10.1002/gps.436.

Douglas, A. N., Binder, K. S., Kajos, J. H., Hyde, J., \& Li, Y. (2013). Reading Relationships, But Seeing Betrayal: Impact of Relational Health Schemas on Processing of Interpersonal Conflict. Journal of Social \& Clinical Psychology, 32(9), 964-988, doi:10.1521/jscp.2013.32.9.964. 
Downey, G., Freitas, A. L., Michaelis, B., \& Khouri, H. (1998). The self-fulfilling prophecy in close relationships: Rejection sensitivity and rejection by romantic partners. Journal of Personality and Social Psychology, 75(2), 545-560, doi:10.1037/00223514.75.2.545.

Duncan, B. L., Miller, S. D., Sparks, J., Claud, D., Reynolds, L., Brown, J., \& Johnson, L. (2003). The Session Rating Scale: Preliminary psychometric properties of a "working" alliance measure. Journal of Brief Therapy, 3(1), 3-12.

Eifert, G. H., \& Heffner, M. (2003). The effects of acceptance versus control contexts on avoidance of panic-related symptoms. Journal of Behavior Therapy and Experimental Psychiatry, 34(3-4), 293-312, doi:10.1016/j.jbtep.2003.11.001.

Elliott, A., Burgio, L., \& DeCoster, J. (2010). Enhancing caregiver health: findings from the Resources for Enhancing Alzheimer's Caregiver Health II intervention. Journal of The American Geriatrics Society, 58(1), 30-37. doi:10.1111/j.15325415.2009.02631.x

Franco, C., Sola, M. D. M., \& Justo, E. (2010). Reducing psychological discomfort and overload in Alzheimer's family caregivers through a mindfulness meditation program. Revista Española de Geriatría y Gerontología, 45(5), 252-258. doi:10.1016/j.regg.2010.03.006.

Gámez, W., Chmielewski, M., Kotov, R., Ruggero, C., Suzuki, N., \& Watson, D. (2014). The brief experiential avoidance questionnaire: Development and initial validation. Psychological Assessment, 26(1), 35-45, doi:10.1037/a0034473.

Gerhart, J. I., Baker, C. N., Hoerger, M., \& Ronan, G. F. (2014). Experiential avoidance and interpersonal problems: A moderated mediation model. Journal of Contextual Behavioral Science, 3(4), 291-298, doi:10.1016/j.jcbs.2014.08.003.

Gray, B., Seddon, D., Robinson, C. A., \& Roberts, A. (2009). An emotive subject: insights from social, voluntary and healthcare professionals into the feelings of family carers for people with mental health problems. Health and Social Care in the Community, 17(2), 125-132, doi:10.1111/j.1365-2524.2008.00803.x.

Grusec, J. E., Hastings, P., \& Mammone, N. (1994). Parenting cognitions and relationship schemas. New Directsions fro Child Development, 66, 5-19. doi: $10.1002 / \mathrm{cd} .23219946603$

Hayes, S. (2004). Acceptance and Commitment Therapy and the New Behavior Therapies: Mindfulness, Acceptance, and Relationship. In Mindfulness and Acceptance: Expanding the Cognitive-behavioral Tradition. New York: Guilford Press. 
Hayes, S., Luoma, J., Bond, F., Masuda, A., \& Lillis, J. (2006). Acceptance and Commitment Therapy: Model, processes and outcomes. Behaviour Research and Therapy, 44(1), 125, doi:10.1016/j.brat.2005.06.006.

Hayes, S., Strosahl, K., \& Wilson, K. G. (1999). Acceptance and Commitment Therapy: An Experiential Approach to Behavior Change. New York: Guilford Press.

Hayes, S., Wilson, K., Gifford, E., Follette, V., \& Strosahl, K. (1996). Experiential avoidance and behavioral disorders: A functional dimensional approach to diagnosis and treatment. Journal of Consulting and Clinical Psychology, 64(6), 1152-1168, doi:10.1002/jclp.20400.

Henderson, J. (2001). 'He's not my carer-he's my husband': personal and policy constructions of care in mental health. Journal of Social Work Practice, 15(2), 149159, doi:10.1080/02650530120090601.

Holtforth, M. G. (2008). Avoidance motivation in psychological problems and psychotherapy. Psychotherapy Research: Journal Of The Society For Psychotherapy Research, 18(2), 147-159, doi:10.1080/10503300701765849.

Holtforth, M. G., Bents, H., Mauler, B., \& Grawe, K. (2006). Interpersonal distress as a mediator between avoidance goals and goal satisfaction in psychotherapy inpatients. Clinical Psychology \& Psychotherapy, 13(3), 172-182, doi:10.1002/cpp.486.

Horowitz, L., Alden, L., Wiggins, J., \& Pincus, A. (2000). IIP, Inventory of Interpersonal Problems Manual. San Antonio, TX: The Psychological Corporation.

Horowitz, L., Rosenberg, S., Baer, B., Ureno, G., \& Villasenor, V. (1988). Inventory of Interpersonal Problems: Psychometric Properties and Clinical Applications. Journal of Consulting and Clinical Psychology, 56(6), 885-892, doi:10.1037/0022006X.56.6.885.

Horowitz, L., Rosenberg, S. E., \& Bartholomew, K. (1993). Interpersonal problems, attachment styles, and outcome in brief dynamic psychotherapy. Journal of Consulting and Clinical Psychology, 61(4), 549-560, doi:10.1037/0022006X.61.4.549.

Inge, B. (1992). The Origins of Attachment Theory: John Bowlby and Mary Ainsworth. Developmental Psychology, 28(5), 759-775, doi:10.1037/0012-1649.28.5.759.

Kopta, S. M. (2003). The dose-effect relationship in psychotherapy: a defining achievement for Dr. Kenneth Howard. Journal of Clinical Psychology, 59(7), 727-733, doi: 10.1002/jclp.10167. 
Laver, K., Milte, R., Dyer, S., \& Crotty, M. (2016). A Systematic Review and Meta-Analysis Comparing Carer Focused and Dyadic Multicomponent Interventions for Carers of People With Dementia. Journal Of Aging And Health, 29(8), 1308-1349. https://doi.org/10.1177/0898264316660414

Lawn, S., \& McMahon, J. (2014). The importance of relationship in understanding the experiences of spouse mental health carers. Qualitative Health Research, 24(2), 254266, doi:10.1177/1049732313520078.

Leoni, M., Corti, S., Cavagnola, R., Healy, O., \& Noone, S. J. (2016). How acceptance and commitment therapy changed the perspective on support provision for staff working with intellectual disability. Advances in Mental Health and Intellectual Disabilities(1), 59-73, doi:10.1108/AMHID-09-2015-0046.

Lev, A. (2011). A new group therapy protocol combining acceptance and commitment therapy (ACT) and Schema Therapy in the treatment of interpersonal disorders: A randomized controlled trial. PhD (Doctor of Philosophy) thesis, Wright Institute, Berkeley, CA.

Levitt, J. T., Brown, T. A., Orsillo, S. M., \& Barlow, D. H. (2004). The Effects of Acceptance Versus Suppression of Emotion on Subjective and Psychophysiological Response to Carbon Dioxide Challenge in Patients With Panic Disorder. Behavior Therapy, 35(4), 747-766, doi:10.1016/S0005-7894(04)80018-2.

Lillis, J., Levin, M. E., \& Hayes, S. C. (2011). Exploring the relationship between body mass index and health-related quality of life: A pilot study of the impact of weight selfstigma and experiential avoidance. Journal of Health Psychology, 16(5), 722-727, doi:10.1177/1359105310388321.

Lindstrom, K. B., \& Melnyk, B. M. (2013). Feasibility and preliminary effects of an intervention targeting schema development for caregivers of newly admitted hospice patients. Journal of Palliative Medicine, 16(6), 680-685, doi:10.1089/jpm.2012.0198.

Losada, A., Márquez-González, M., Romero-Moreno, R., \& López, J. (2014). Development and validation of the Experiential Avoidance in Caregiving Questionnaire (EACQ). Aging and Mental Health, 18(7), 897-904, doi:10.1080/13607863.2014.896868.

Losada, A., Márquez-González, M., Romero-Moreno, R., López, J., Fernández-Fernández, V., \& Nogales-González, C. (2015). Attending to dementia caregivers diverse needs: Contributions from cognitive behavioral therapy and acceptance and commitment therapy. Clínica y Salud, 26(1), 41-48, doi:10.1016/j.clysa.2015.02.001. 
Mandavia, A., Masuda, A., Moore, M., Mendoza, H., Donati, M. R., \& Cohen, L. L. (2015). Empirical Research: The application of a cognitive defusion technique to negative body image thoughts: A preliminary analogue investigation. Journal of Contextual Behavioral Science, 4, 86-95, doi:10.1016/j.jcbs.2015.02.003.

Manne, S. L., Ostroff, J. S., Norton, T. R., Fox, K., Goldstein, L., \& Grana, G. (2006). Cancer-related relationship communication in couples coping with early stage breast cancer. Psycho-Oncology, 15(3), 234-247, doi:10.1002/pon.941.

Masuda, A., Twohig, M. P., Stormo, A. R., Feinstein, A. B., Chou, Y.-Y., \& Wendell, J. W. (2010). The effects of cognitive defusion and thought distraction on emotional discomfort and believability of negative self-referential thoughts. Journal of Behavior Therapy and Experimental Psychiatry, 41, 11-17, doi:10.1016/j.jbtep.2009.08.006.

McConachie, D. A. J., McKenzie, K., Morris, P. G., \& Walley, R. M. (2014). Acceptance and mindfulness-based stress management for support staff caring for individuals with intellectual disabilities. Research in Developmental Disabilities, 35(6), 1216-1227, doi:10.1016/j.ridd.2014.03.005.

McKay, M., Lev, A., \& Skeen, M. (2012). Acceptance and Commitment Therapy for Interpersonal Problems: Using Mindfulness, Acceptance, and Schema Awareness to Change Interpersonal Behaviors: New Harbinger Publications.

Miller, S. D., Duncan, B. L., Brown, J., Sparks, J., \& Claud, D. (2003). The Outcome Rating Scale: A preliminary study of the reliability, validity, and feasibility of a brief visual analog measure. Journal of Brief Therapy, 2, 91-100.

Millstein, D. J., Orsillo, S. M., Hayes-Skelton, S. A., \& Roemer, L. (2015). Interpersonal Problems, Mindfulness, and Therapy Outcome in an Acceptance-Based Behavior Therapy for Generalized Anxiety Disorder. Cognitive Behaviour Therapy, 44(6), 491501, doi:10.1080/16506073.2015.1060255.

Moore, T., \& McArthur, M. (2007). We're all in it together: supporting young carers and their families in Australia. Health \& Social Care in the Community, 15(6), 561-568, doi: 10.1111/j.1365-2524.2007.00719.x.

Noone, S., \& Hastings, R. (2010). Using Acceptance and Mindfulness-Based Workshops with Support Staff Caring for Adults with Intellectual Disabilities. Mindfulness, 1(2), 67-73, doi:10.1007/s 12671-010-0007-4.

Noone, S., \& Hastings, R. (2011). Values and Psychological Acceptance as Correlates of Burnout in Support Staff Working With Adults With Intellectual Disabilities. Journal 
of Mental Health Research in Intellectual Disabilities, 4(2), 79-89, doi:10.1080/19315864.2011.582230.

Oyebode, J. (2003). Assessment of carers' psychological needs. Advances in Psychiatric Treatment, 9(1), 45-53, doi:10.1192/apt.9.1.45.

Rayan, A., \& Ahmad, M. (2017). Mindfulness and parenting distress among parents of children with disabilities: A literature review. Perspectives in Psychiatric Care, 1, 17, doi:10.1111/ppc.12217.

Renner, F., Jarrett, R. B., Vittengl, J. R., Barrett, M. S., Clark, L. A., \& Thase, M. E. (2012). Interpersonal problems as predictors of therapeutic alliance and symptom improvement in cognitive therapy for depression. Journal of Affective Disorders, 138(3), 458-467, doi:10.1016/j.jad.2011.12.044.

Sadath, A., Muralidhar, D., Varambally, S., \& Gangadhar, B. N. (2017). Does group intervention have benefits on expressed emotion and social support in carers of persons with first episode psychosis? Social Work in Mental Health, 1-14, doi:10.1080/15332985.2016.1252826.

Sadler, E., \& McKevitt, C. (2013). 'Expert carers': An emergent normative model of the caregiver. Social Theory \& Health, 11(1), 40-58, doi:http://dx.doi.org/10.1057/sth.2012.17.

Salzer, S., Winkelbach, C., Leibing, E., Pincus, A. L., \& Leichsenring, F. (2011). Interpersonal subtypes and change of interpersonal problems in the treatment of patients with generalized anxiety disorder: A pilot study. Psychotherapy, 48(3), 304310, doi:10.1037/a0022013.

Scott, J. L., Dawkins, S., Quinn, M. G., Sanderson, K., Elliott, K. J., Stirling, C ... Robinson, A. (2016). Caring for the carer: A systematic review of pure technology-based cognitive behavioral therapy (TB-CBT) interventions for dementia carers. Aging and Mental Health, 20(8), 793-803, doi:10.1080/13607863.2015.1040724.

Spector, A., Charlesworth, G., Orrell, M., \& Marston, L. (2016). Factors influencing the person-carer relationship in people with anxiety and dementia. Aging and Mental Health, 20(10), 1055-1062, doi:10.1080/13607863.2015.1063104.

Stulz, N., Lutz, W., Kopta, S. M., Minami, T., \& Saunders, S. M. (2013). Dose-effect relationship in routine outpatient psychotherapy: Does treatment duration matter? Journal of Counseling Psychology, 60(4), 593-600, doi:10.1037/a003358910.1037/a0033589.supp 
Sullivan, H. S. (1953). The Interpersonal Theory of Psychiatry. New York: Norton.

Thimm, J. C. (2013). Early maladaptive schemas and interpersonal problems: A circumplex analysis of the YSQ-SF. International Journal of Psychology \& Psychological Therapy, 13(1), 113-124.

Ulstein, I., Wyller, T. B., \& Engedal, K. (2008). Correlates of intrusion and avoidance as stress response symptoms in family carers of patients suffering from dementia. International Journal of Geriatric Psychiatry, 23(10), 1051-1057, doi:10.1002/gps.2032.

Wadham, O., Simpson, J., Rust, J., \& Murray, C. (2016). Couples' shared experiences of dementia: A meta-synthesis of the impact upon relationships and couplehood. Aging and Mental Health, 20(5), 463-473, doi:10.1080/13607863.2015.1023769.

Weinrib, A. Z. (2011). Investigating experiential avoidance as a mechanism of action in a mindfulness intervention. $\mathrm{PhD}$ (Doctor of Philosophy) thesis, University of Iowa.

Wilkinson, C., \& McAndrew, S. (2008). 'I'm not an outsider, I'm his mother!' A phenomenological enquiry into carer experiences of exclusion from acute psychiatric settings. International Journal of Mental Health Nursing, 17(6), 392-401, doi:10.1111/j.1447-0349.2008.00574.x.

Williams, J. C., \& Lynn, S. J. (2010). Acceptance: An historical and conceptual review. Imagination, Cognition and Personality, 30(1), 5-56, doi:10.2190/IC.30.1.c.

Yesufu-Udechuku, A., Harrison, B., Mayo-Wilson, E., Young, N., Woodhams, P., Shiers, D ... Kendall, T. (2015). Interventions to improve the experience of caring for people with severe mental illness: Systematic review and meta-analysis. British Journal of Psychiatry, 206(4), 268-274, doi:10.1192/bjp.bp.114.147561.

Young, J., Klosko, J., \& Weishaar, M. (2006). Schema Therapy: A Practitioner's Guide: Guilford Publications.

Young, T. J., Manthorp, C., Howells, D., \& Tullo, E. (2011). Developing a carer communication intervention to support personhood and quality of life in dementia. Ageing and Society, 31, 1003-1025, doi:10.1017/S0144686X10001182 
Table 1.

Structure and components of the Me and My Relationships program

\begin{tabular}{|c|c|c|c|}
\hline Phase & Week & Session content & Example exercises \\
\hline \multirow[t]{4}{*}{$\begin{array}{l}\text { Understanding } \\
\text { current patterns }\end{array}$} & Week 1 & Icebreaker, orientation, group rules, introductions to schemas and mindfulness & $\begin{array}{l}\text { Mindful breathing; psychoeducation on common } \\
\text { types of schematic thoughts }\end{array}$ \\
\hline & Week 2 & Mindfulness, review schemas, my current and ideal relationships & Mindful eating; completion of eco-map \\
\hline & Week 3 & Mindfulness, schema questionnaire; identifying my patterns & $\begin{array}{l}\text { Focusing using our } 5 \text { senses; review personal } \\
\text { experiences with schemas }\end{array}$ \\
\hline & Week 4 & Mindfulness, schema coping behaviours, costs and benefits of common coping behaviors & Psychoeducation on common coping behaviors \\
\hline \multirow{3}{*}{$\begin{array}{l}\text { Transforming } \\
\text { current patterns }\end{array}$} & Week 6 & Mindfulness, value clarification, setting values-oriented goals, barriers; willingness & $80^{\text {th }}$ birthday visualization; Monsters on the bus \\
\hline & Week 7 & Mindfulness, review and set new goals, defusion psychoeducation and practice & Salesperson metaphor; Leaves on the stream \\
\hline & Week 8 & Review and set new goals, self-evaluations and fusion, self as observer, mindfulness & Activity: evaluations vs descriptions \\
\hline \multirow{2}{*}{$\begin{array}{l}\text { Strengthening } \\
\text { new patterns }\end{array}$} & Week 10 & $\begin{array}{l}\text { Review and set new goals, workability of emotional control, defusion, self as observer, } \\
\text { mindfulness }\end{array}$ & Hungry lion metaphor, Naming the story \\
\hline & Week 11 & $\begin{array}{l}\text { Review and set new goals, effective communication, acceptance, termination preparation, } \\
\text { mindfulness }\end{array}$ & $\begin{array}{l}\text { Communication role-play; Loving kindness } \\
\text { meditation }\end{array}$ \\
\hline
\end{tabular}


Table 2

Means, standard deviations and repeated measures ANOVA's over time $(n=24)$

\begin{tabular}{|c|c|c|c|c|c|c|c|}
\hline \multirow[t]{2}{*}{ Measures } & \multicolumn{2}{|c|}{ Week 1} & \multicolumn{2}{|c|}{ Week 6} & \multicolumn{2}{|c|}{ Week 12} & \multirow{2}{*}{$\begin{array}{c}\text { F value } \\
(\mathrm{df}=2,46)\end{array}$} \\
\hline & Mean & SD & Mean & SD & Mean & SD & \\
\hline Total Interpersonal Problems & $61.88^{\mathrm{a}}$ & 11.19 & 56.92 & 10.93 & $53.33^{\mathrm{a}}$ & 8.58 & $6.63^{* *}$ \\
\hline Domineering/Controlling & 53.58 & 12.29 & $52.00^{\mathrm{a}}$ & 9.35 & $48.54^{\mathrm{a}}$ & 8.28 & $4.64^{*}$ \\
\hline Vindictive/Self-Centered & 55.25 & 13.68 & 50.50 & 8.56 & 49.25 & 8.12 & $2.51 \#$ \\
\hline Cold/Distant & $57.21^{\mathrm{a}}$ & 14.26 & 52.50 & 10.44 & $50.54^{\mathrm{a}}$ & 9.52 & $4.75^{*}$ \\
\hline Socially Inhibited & 56.96 & 13.16 & 53.33 & 12.02 & 51.21 & 10.96 & 2.28 \\
\hline Non-assertive & $62.83^{\mathrm{a}}$ & 11.76 & 58.04 & 12.29 & $54.54^{\mathrm{a}}$ & 10.32 & $5.13^{*}$ \\
\hline Overly Accommodating & $64.38^{\mathrm{ab}}$ & 12.58 & $57.54^{\mathrm{a}}$ & 11.01 & $56.00^{\mathrm{b}}$ & 10.39 & $8.21^{* *}$ \\
\hline Self-Sacrificing & 61.67 & 11.82 & 59.42 & 8.99 & 55.83 & 8.49 & $3.81^{*}$ \\
\hline Intrusive/Needy & 55.04 & 11.12 & 55.25 & 11.66 & 52.54 & 9.90 & 0.87 \\
\hline Experiential avoidance & $54.17^{\mathrm{a}}$ & 13.00 & 49.00 & 11.90 & $44.13^{\mathrm{a}}$ & 9.50 & $10.21^{* * *}$ \\
\hline Caregiving avoidance & $39.63^{\mathrm{a}}$ & 8.03 & $37.13^{\mathrm{b}}$ & 7.35 & $32.00^{\mathrm{ab}}$ & 6.55 & $17.07^{* * *}$ \\
\hline Mindfulness & $48.00^{\mathrm{ab}}$ & 12.75 & $55.46^{\mathrm{a}}$ & 12.87 & $58.13^{\mathrm{b}}$ & 11.40 & $13.75^{* *}$ \\
\hline Wellbeing & $19.98^{\mathrm{ab}}$ & 7.77 & $25.19^{\mathrm{bc}}$ & 8.78 & $30.79^{\mathrm{ac}}$ & 6.45 & $23.92 * * *$ \\
\hline
\end{tabular}

Note.

${ }^{*} \mathrm{p}<.05,{ }^{* *} \mathrm{p}<.01,{ }^{* * *} \mathrm{p}<.001$

${ }^{\text {abc }}$ Means that share a superscript in each row are significantly different at $\mathrm{p}<.05$ (Bonferroni adjusted)

\# Greenhouse Geisser adjustment ( $\mathrm{df}=1.51,34.61)$ 
Table3.

Proportions of individuals experiencing above average $(\geq 60)$ interpersonal problems at different points during the intervention $(n=24)$

\begin{tabular}{llll}
\hline & Week 1 & Week 6 & Week 12 \\
& $\%(\mathrm{~N})$ & $\%(\mathrm{~N})$ & $\%(\mathrm{~N})$ \\
\hline Domineering/Controlling & $16.7 \%(4)$ & $25.0 \%(6)$ & $8.3 \%(2)$ \\
Vindictive/Self-Centered & $25.0 \%(6)$ & $12.5 \%(3)$ & $8.3 \%(2)$ \\
Cold/Distant & $37.5 \%(9)$ & $20.8 \%(5)$ & $16.7 \%(4)$ \\
Socially Inhibited & $41.7 \%(10)$ & $29.2 \%(7)$ & $20.8 \%(5)$ \\
Non-assertive & $58.3 \%(14)$ & $41.7 \%(10)$ & $20.8 \%(5)$ \\
Overly Accommodating & $62.5 \%(15)$ & $37.5 \%(9)$ & $29.2 \%(7)$ \\
Self-Sacrificing & $66.7 \%(16)$ & $54.2 \%(13)$ & $25.0 \%(6)$ \\
Intrusive/Needy & $29.2 \%(7)$ & $25.0 \%(6)$ & $12.5 \%(3)$ \\
Total Interpersonal Problems & $58.3 \%(14)$ & $33.3 \%(8)$ & $16.7 \%(4)$ \\
\hline
\end{tabular}


Table 4. Themes and subthemes of focus group feedback on the Me and My Relationship program

\begin{tabular}{|c|c|c|c|}
\hline $\begin{array}{l}\text { Themes and } \\
\text { subthemes }\end{array}$ & $\begin{array}{l}\text { Prevalence } \\
(\%)^{\mathrm{a}}\end{array}$ & $\begin{array}{l}\text { Frequency } \\
(\mathrm{N})^{\mathrm{b}}\end{array}$ & Illustrating examples ${ }^{\mathrm{c}}$ \\
\hline $\begin{array}{l}\text { 1. Aspects of } \\
\text { program }\end{array}$ & $100 \%$ & 26 & \\
\hline $\begin{array}{l}\text { a. Program } \\
\text { content }\end{array}$ & $67 \%$ & 15 & $\begin{array}{l}\text { 'The most helpful parts were the schemas- the things that drive your behaviour. The } \\
\text { mindfulness, because it's key to me for separation of myself and feelings' (1.1) } \\
\text { 'For me the biggest thing was values, the core values right down to the person that you } \\
\text { want to be- and the question 'am I living by those values' (1.3) } \\
\text { 'Mindfulness has helped me a lot' (3.1) }\end{array}$ \\
\hline b. Relational & $67 \%$ & 11 & $\begin{array}{l}\text { 'The facilitators were very caring, I felt like if I needed to talk I could talk' (3.2) } \\
\text { 'You learn that you're not alone' (1.4) } \\
\text { 'One of the other group members also had a daughter. I heard my story a bit, and how } \\
\text { she deals with it. It made me realize that I need to let go a bit more' (2.2) }\end{array}$ \\
\hline $\begin{array}{l}\text { 2. Changes in } \\
\text { Emotion }\end{array}$ & $92 \%$ & 22 & \\
\hline $\begin{array}{ll}\text { a. } & \text { Reduced } \\
& \text { frequency } \\
& \text { and } \\
& \text { intensity }\end{array}$ & & & $\begin{array}{l}\text { 'I asked my wife and my daughter whether they'd noticed any changes since the course. } \\
\text { My wife said that I'm less stressed and less angry. My daughter said I'm not as much of } \\
\text { an emotional pushover' (1.1) } \\
\text { 'I lived with a lot of guilt... I don't live with that guilt anymore' (2.2) } \\
\text { 'My anxiety is not as bad as it used to be' (3.4) }\end{array}$ \\
\hline $\begin{array}{l}\text { b. Relationsh } \\
\text { ip with }\end{array}$ & & & $\begin{array}{l}\text { 'I sit back and feel the feelings, and practice feeling them. I try to be the sky, and I know } \\
\text { that they are not really me' (2.3) }\end{array}$ \\
\hline
\end{tabular}




\begin{tabular}{|c|c|c|c|}
\hline emotions & & & $\begin{array}{l}\text { 'I've got the ability to see it over there, leaves going down the river in the mindfulness } \\
\text { sense, I can separate my emotions from who I am and what's important to me' (3.3) } \\
\text { 'I'm not as afraid to address my feelings and think about it' (1.4) }\end{array}$ \\
\hline 3. Reactivity & $83 \%$ & 23 & $\begin{array}{l}\text { 'I respond more. I’ve learnt not to react so much’ (1.1) } \\
\text { 'I didn’t realize that the schemas caused me to react in the wrong way. It’s made me } \\
\text { realise how I was reacting was not good, and that I had to change as well’ (2.1) } \\
\text { 'Being able to manage how you react and respond to how you're feeling, rather than } \\
\text { going into this chaotic emotional drama' (2.4) }\end{array}$ \\
\hline $\begin{array}{l}\text { 4. Acceptance of } \\
\text { caregiving } \\
\text { situation }\end{array}$ & $75 \%$ & 18 & $\begin{array}{l}\text { 'With my son I have accepted that that's his diagnosis, he’s not going to change... I’ve } \\
\text { got a lot more ability to accept things and say 'you can’t change it' (1.4) } \\
\text { 'Realizing that's it life and it's not going to go away' (2.3) } \\
\text { 'I have more understanding about my daughter, I accept it. When things are difficult, I } \\
\text { go ahead more than before. Whatever comes I try to manage it' (3.1) }\end{array}$ \\
\hline 5. Communication & $67 \%$ & 18 & $\begin{array}{l}\text { 'I've learnt to listen closer to what my son says- actively listen- whereas before I would } \\
\text { just go into my panic stage and not really hear it' (1.2) } \\
\text { 'My husband is actually listening more and taking advice, whereas before he would just } \\
\text { shut off' (3.4) } \\
\text { 'Not as much fired up communication as before. It's more the quality of conversation } \\
\text { and understanding now' (3.2) }\end{array}$ \\
\hline 6. Agency & $58 \%$ & 14 & \\
\hline a. Assertiven & $50 \%$ & 8 & 'I have tried to be more assertive. I have the strength now to say I have had enough, you \\
\hline
\end{tabular}




\begin{tabular}{|c|c|c|c|}
\hline ess & & & $\begin{array}{l}\text { need to stop. I feel stronger in myself' (3.3) } \\
\text { 'I learnt how to actually be assertive about things and not aggressive when I wanted to } \\
\text { say something' (1.3) } \\
\text { 'For me it's taught me to be a bit more assertive, whereas before I would just do the run- } \\
\text { away. I'm finding I'm not doing that, I'm actually voicing my opinion' (1.2) }\end{array}$ \\
\hline b. Control & $25 \%$ & 4 & $\begin{array}{l}\text { 'I'm coping a lot better at letting difficult situations be, without having to run up there in } \\
\text { person and take control of everyone and everything' (3.2) } \\
\text { 'I'm learning when to step in and when to step out' (2.3) } \\
\text { 'I don't push him anymore now, I allow him to make the choice' (1.2) }\end{array}$ \\
\hline 7. Connection & $58 \%$ & 13 & $\begin{array}{l}\text { 'My relationship with my son is great, we're getting on great, he's posting things on } \\
\text { Facebook like “I've got the greatest mum ever”. A year ago if you’d said that it would } \\
\text { have been “I wanna kill my mum” (1.4) } \\
\text { 'Now I feel like I'm living by my values, being the mum I want to be, being the friend I } \\
\text { want to be, being the wife I want to be' (2.4) }\end{array}$ \\
\hline
\end{tabular}

\footnotetext{
${ }^{\mathrm{a}}$ Prevalence (\%) of participants who discussed this theme

${ }^{\mathrm{b}}$ Number of references to this theme

${ }^{\mathrm{c}}$ Quotes from participants are coded according to focus group attended (range of 1-3) and identifying number within that focus group (range of 1-4).
} 